\title{
Notations and conventions in molecular spectroscopy: part 3. Permutation and permutation-inversion symmetry notation
}

Article

Published Version

Bunker, P. R., Schutte, C. J. H., Hougen, J. T., Mills, I., Watson, J. K. G. and Winnewisser, B. P. (1997) Notations and conventions in molecular spectroscopy: part 3. Permutation and permutation-inversion symmetry notation. Pure and Applied Chemistry, 69 (8). pp. 1651-1657. ISSN 0033-4545 Available at https://centaur.reading.ac.uk/7303/

It is advisable to refer to the publisher's version if you intend to cite from the work. See Guidance on citing.

Published version at: http://old.iupac.org/publications/pac/1997/6908/index.html

Publisher: International Union of Pure and Applied Chemistry

All outputs in CentAUR are protected by Intellectual Property Rights law, including copyright law. Copyright and IPR is retained by the creators or other copyright holders. Terms and conditions for use of this material are defined in the End User Agreement.

www.reading.ac.uk/centaur 
Central Archive at the University of Reading

Reading's research outputs online 


\title{
INTERNATIONAL UNION OF PURE AND APPLIED CHEMISTRY
}

\author{
PHYSICAL CHEMISTRY DIVISION \\ COMMISSION ON MOLECULAR STRUCTURE AND SPECTROSCOPY* \\ SUBCOMMITTEE ON NOTATIONS AND CONVENTIONS FOR MOLECULAR SPECTROSCOPY
}

\section{NOTATIONS AND CONVENTIONS IN MOLECULAR SPECTROSCOPY: PART 3. PERMUTATION AND PERMUTATION-INVERSION SYMMETRY NOTATION}

(IUPAC Recommendations 1997)

\author{
Prepared for publication by \\ P. R. BUNKER ${ }^{1}$, C. J. H. SCHUTTE ${ }^{2}$, J. T. HOUGEN ${ }^{3}$, I. M. MILLS ${ }^{4}$, J. K. G. WATSON ${ }^{1}$ AND \\ B. P. WINNEWISSER ${ }^{5}$
}

\begin{abstract}
${ }^{1}$ Steacie Institute for Molecular Sciences, National Research Council of Canada, Ottawa, K1A OR6, Canada ${ }^{2}$ University of South Africa, PO Box 392, Pretoria 0001, South Africa

${ }^{3}$ Molecular Physics Division, National Institute for Standards and Technology, Gaithersburg, Maryland 20899, USA

${ }^{4}$ Department of Chemistry, University of Reading, Whiteknights, Reading, RG6 2AD, UK

${ }^{5}$ Justus Liebig Universität, Physikalisch-Chemisches Institut, Heinrich-Buff-Ring 58, D-6300 Giessen, Germany
\end{abstract}

\begin{abstract}
*Membership of the Commission during the preparation of this report (1985-97) was as follows:
Chairman: J. R. Durig (USA; 1983-89); C. J. H. Schutte (RSA; 1989-93); J. E. Bertie (Canada; 1994-97); Secretary: H. A. Willis (UK; 1983-87); J. G. Grasselli (USA; 1987-89); J. F. Sullivan (USA; 1989-95); P. Klaeboe (Norway; 1996-97); Titular Members: A. M. Bradshaw (FRG; 1983-91); R. D. Brown (Australia; 1987-93); B. G. Derendjaev (Russia; 1983-91); R. K. Harris (UK; 1989-93); A. M. Heyns (RSA; 1994-97); E. Hirota (Japan; 1985-93); R. Janoschek (Austria; 1994-97); P. Klaeboe (Norway; 1994-97); J. F. J. Todd (UK; 1981-89); S. Tsuchiya (Japan; 1994-97); H. A. Willis (UK; 1983-87); B. P. Winnewisser (FRG; 1991-97); Associate Members: A. M. Bradshaw (FRG; 1991-95); S. M. Cabral de Menezes (Brazil; 1994-97); B. G. Derendjaev (Russia; 1991-95); P. Granger (France; 1989-93); R. K. Harris (UK; 1981-89); E. Hirota (Japan; 1994-97); J. Kowalewski (Sweden; 1996-97); S. Leach (France; 1987-91); G. J. Martin (France; 1983-89); J. P. Maier (Switzerland; 1989-93); L. N. Masalov (Russia; 1987-91); A. Oskam (Netherlands; 1991-97); W. B. Person (USA; 1983-89); C. J. H. Schutte (RSA; 1983-89); J. F. J. Todd (UK; 1989-91); S. Tsuchiya (Japan; 1987-93); C. Zhang (China; 1989-97); National Representatives: J. E. Collin (Belgium; 1985-97); S. M. Cabral de Menezes (Brazil; 1991-93); S. L. Spassov (Bulgaria; 1987-93); J. Lu (China; 1981-89); E. Hadjoudis (Greece; 1991-93); G. Varsányi (Hungary; 1985-93); M. Chowdhury (India; 1986-95); Z. Luz (Israel; 1983-93); S. Califano (Italy; 1989-97); S. Ng (Malaysia; 1983-93); A. Oskam (Netherlands; 1989-91); B. Jezowska-Trzebiatowska (Poland; 1983-89); J. J. C. Teixeira-Dias (Portugal; 1991-97); Y. S. Lee (Korea; 1989-97); T. A. Ford (RSA; 1987-91); C. Pascual (Spain; 1987-91); D. Escolar (Spain; 1991-97); J. Kowalewski (Sweden; 1994-95); M. Cebe (Turkey; 1987-91); S. Suzer (Turkey; 1991-93, 1995-97); S. Içli (Turkey; 1993-95); T. Ast (Yugoslavia; 1989-93); R. K. Harris (UK; 1993-97).
\end{abstract}

Republication or reproduction of this report or its storage and/or dissemination by electronic means is permitted without the need for formal IUPAC permission on condition that an acknowledgement, with full reference to the source along with use of the copyright symbol $(\mathcal{C}$, the name IUPAC and the year of publication are prominently visible. Publication of a translation into another language is subject to the additional condition of prior approval from the relevant IUPAC National Adhering Organization. 


\title{
Notations and conventions in molecular spectroscopy: Part 3. Permutation and permutation-inversion symmetry notation (IUPAC Recommendations 1997)
}

\begin{abstract}
The field of Molecular Spectroscopy was surveyed in order to determine a set of conventions and symbols which are in common use in the spectroscopic literature. This document, which is Part 3 in a series, deals with symmetry notation referring to groups that involve nuclear permutations and the inversion operation. Further parts will follow, dealing inter alia with vibration-rotation spectroscopy and electronic spectroscopy.
\end{abstract}

\section{PREAMBLE}

The sub-committee on Notations and Conventions in Molecular Spectroscopy was initiated by Commission I.5, the Commission on Molecular Structure and Spectroscopy, to establish and recommend a set of conventions and symbols from those that are in common use in the spectroscopic literature.

Part 1 of the series is titled General Spectroscopic Notation [1], and Part 2 is titled Symmetry Notation [2]. The present part deals with symmetry notation referring to groups that involve nuclear permutations and the inversion operation. The recommendations follow the notation of Bunker [3], and in particular the symmetry groups involved will be called Molecular Symmetry Groups after the work of Longuet-Higgins [4].

Part 3.

\section{PERMUTATION AND PERMUTATION-INVERSION SYMMETRY NOTATION}

\author{
3.1 General Comments \\ 3.2 Symmetry Operations for the Molecular Symmetry Groups \\ 3.3 The Molecular Symmetry Groups \\ 3.4 Representations for Molecular Symmetry Groups \\ Appendix of Examples
}

\subsection{GENERAL COMMENTS}

For a given molecule the group consisting of the identity and all permutations of the space and spin coordinates of identical nuclei is called its Complete Nuclear Permutation Group. The direct product of the Complete Nuclear Permutation Group with the group $\left\{E, E^{*}\right\}$, where $E$ is the identity and $E^{*}$ the inversion of the space coordinates of all nuclei and electrons in the molecular centre of mass, is called its Complete Nuclear Permutation Inversion Group.

The subgroup of the Complete Nuclear Permutation Inversion Group of a molecule, obtained by deleting all unfeasible elements, is called its Molecular Symmetry Group; the elements in the Molecular Symmetry Group are said to be feasible. An unfeasible element is one that interconverts nuclear-numbered equilibrium forms of the molecule when these forms are separated by an insuperable barrier in the potential energy surface; an insuperable barrier is one that does not allow observable tunnelling to occur through it at the energy resolution of the experiment being performed (i.e., no energy level shifts or splittings resulting from tunnelling are detected). Tunnelling may not be observable if we use a low resolution experiment yet it may be apparent if we use a high resolution experiment; the Molecular Symmetry Group that we use to analyze the results will then be different for the two cases since the elements associated with the tunnelling are feasible in the latter (high resolution) case but not in the former (low resolution) case. 
For molecules that have a non-linear equilibrium configuration, and for which there are no observable effects on the energy levels from tunnelling motions, the Molecular Symmetry Group is isomorphic to the Molecular Point Group and each element of the Molecular Symmetry Group has the same effect on the vibronic coordinates as its partner in the Molecular Point Group. In general, the Molecular Symmetry Group is not isomorphic to any point group. For linear molecules, and molecules having coaxial internal rotors and observable tunnelling effects, it is sometimes useful to use a group of higher order than the Molecular Symmetry Group (see, Hougen [5] and Bunker and Papoušek [6]), and such a group is called an Extended Molecular Symmetry Group.

\subsection{SYMMETRY OPERATIONS FOR THE MOLECULAR SYMMETRY GROUPS}

3.2.1 Identity operation $E$

3.2.2 The pair permutation (or exchange) of the space and spin coordinates of identical nuclei labelled $i$ and $j$

3.2.3 The cyclic permutation of the space and spin coordinates of identical nuclei labelled $i, j, k, \ldots, m, n$ where nucleus $i$ is replaced by nucleus $j, j$ by $k, \ldots, m$ by $n$, and $n$ by $i$. After such an operation the space and spin coordinates of nucleus $j$ will be the same as those that nucleus $i$ had initially, those of $k$ will be the same as those that $j$ had initially, ..., those of $n$ will be the same as those that $m$ had initially, and those of $i$ will be the same as those that $n$ had initially.

3.2.4 The inversion of the space coordinates of all nuclei and electrons of the molecule in the molecular centre of mass

$E^{*}$

$(i j k \ldots m n)^{*}$ inversion $E^{*}$

3.2.6 The operation of rotating one internal rotor through $2 \pi$ radians in a molecule with coaxial internal rotors for which it is necessary to use an Extended Molecular Symmetry Group.

$E^{\prime}$

3.2.7 Operations of the Extended Molecular Symmetry Group of a linear molecule involving a change in the Euler angle $\chi$ of the isomorphic Hamiltonian [3,6]

\subsection{THE MOLECULAR SYMMETRY GROUPS}

3.3.1 For a non-linear molecule with no observable tunnelling effects the Molecular Symmetry Group is isomorphic to the Molecular Point Group, and we recommend that the same symbol be used for it; in cases where it is necessary to distinguish the Molecular Symmetry Group from the Molecular Point Group we recommend adding $(\mathrm{M})$ after the Point Group name, e.g. $C_{2 \mathrm{v}}(\mathrm{M})$ for the Molecular Symmetry Group of the water molecule. For linear molecules with no observable tunnelling effects the symbol for the Molecular Symmetry Group should be $C_{\infty \mathrm{v}}(\mathrm{M})$ or $D_{\infty \mathrm{h}}(\mathrm{M})$ and the symbol for the Extended Molecular Symmetry Group should be $C_{\text {oov }}(\mathrm{EM})$ or $D_{\infty \mathrm{h}}(\mathrm{EM})$ as the Molecular Point Group is $C_{\infty \mathrm{V}}$ or $D_{\infty \mathrm{h}}$, respectively. 
3.3.2 The Molecular Symmetry Group of a tunnelling molecule having order $n$. To distinguish between non-isomorphic groups having the same number of elements, no accepted general convention has yet emerged.

$G_{n}$

$G_{n}^{(m)}(\mathrm{EM})$

\subsection{REPRESENTATIONS FOR MOLECULAR SYMMETRY GROUPS}

3.4.1 For a non-tunnelling non-linear molecule the same notation for the irreducible representations is to be used as in the isomorphic Molecular Point Group.

3.4.2 For a tunnelling molecule the conventions of 2.3.1, 2.3.2, $2.3 .4,2.3 .10$ and 2.3.12 of [2] apply. One-dimensional representations are labelled $\mathrm{A}$ or $\mathrm{B}$, two-dimensional representations are labelled E following 2.3.3 (c) of [2], and recommendations 2.3.3 (d)-(f) of [2] also apply for the labelling of higher dimensioned representations.

\section{REFERENCES}

1 C. J. H. Schutte, J. E. Bertie, P. R. Bunker, J. T. Hougen, I. M. Mills, J. K. G. Watson and B. P. Winnewisser,(Part 1), Pure Appl. Chem., this issue (1997).

2 C. J. H. Schutte, J. E. Bertie, P. R. Bunker, J. T. Hougen, I. M. Mills, J. K. G. Watson and B. P. Winnewisser,(Part 2), Pure Appl. Chem., this issue (1997).

3 P. R. Bunker, Molecular Symmetry and Spectroscopy, Academic Press, New York, 1979.

4 H. C. Longuet-Higgins, Mol. Phys., 6 (1963) 445.

5 J. T. Hougen, Can. J. Phys., 42 (1964) 1920.

6 P. R. Bunker and D. Papoušek, J. Mol. Spectrosc., 32 (1969) 419. 


\section{APPENDIX OF EXAMPLES}

\section{The methane molecule}

The methane molecule is an example of a nonlinear molecule for which there is no observable tunnelling (i.e. no inversion splittings are seen in the spectrum). We number the protons 1 through 4 and the Complete Nuclear Permutation Group consists of all possible proton permutations; this group has $4 !=24$ elements. The Complete Nuclear Permutation Inversion Group consists of $4 ! \times 2=48$ elements (see Table I). Because the effects of inversion tunnelling are not observed in the spectrum, elements such as $E^{*},(12)$, (1423), (123)* and (14)(23)* are unfeasible; there are 24 unfeasible elements. Removing these 24 unfeasible elements from the Complete Nuclear Permutation Inversion Group gives the Molecular Symmetry Group of methane. This group of 24 elements is isomorphic to the Molecular Point Group $T_{\mathrm{d}}$ and is called $T_{\mathrm{d}}(\mathrm{M})$ (see Table II).

\section{The ethane molecule}

The ethane molecule is an example of a molecule for which tunnelling effects are observed. We label the carbon nuclei 7 and 8, the three protons attached to $\mathrm{C}-7$ are labelled $\mathrm{H}-1, \mathrm{H}-2$ and $\mathrm{H}-3$, and the three protons attached to $\mathrm{C}-8$ are labelled $\mathrm{H}-4, \mathrm{H}-5$ and $\mathrm{H}-6$. The Complete Nuclear Permutation Inversion Group consists of $2 ! \times 6 ! \times 2=2880$ elements. The molecule has a staggered equilibrium configuration but the effects of torsional tunnelling are observed. Thus, while elements such as (12), (78), (14) and $E^{*}$ are unfeasible, elements such as (123) and (456) are feasible. There are 36 feasible elements and these constitute the Molecular Symmetry Group of ethane; the group is called $G_{36}$ (see Table III). If we wish to classify the rotational and torsional wavefunctions separately then it is necessary to use the Extended Molecular Symmetry Group $G_{36}\left(\mathrm{EM}\right.$ ) (see Table A-25 of ref. 3). Neither $G_{36}$ nor $G_{36}(\mathrm{EM}$ ) is isomorphic to a point group.

\section{The carbon dioxide molecule}

The carbon dioxide molecule is an example of a linear molecule. Labelling the oxygen nuclei 1 and 2 the Complete Nuclear Permutation Inversion Group is the group of four elements $\left\{E,(12), E^{*},(12)^{*}\right\}$. These are all feasible elements and thus the Molecular Symmetry Group also consists of these four elements (see Table IV). This group is not isomorphic to the Molecular Point Group $D_{\infty \mathrm{ch}}$. If we wish to classify the rotational and vibronic wavefunction separately, it is necessary to use the Extended Molecular Symmetry Group $D_{\infty \mathrm{h}}(\mathrm{EM})$ which is isomorphic to the Molecular Point Group $D_{\infty \mathrm{h}}$ (see Table V). In the group $D_{\infty \mathrm{h}}(\mathrm{EM})$ the operation $(12)_{\pi}^{*}$ has the same effect on the vibronic variables as the point group operation $i$, and the operation $(12)_{\pi+\varepsilon}^{*}$ has the same effect as the point group operation $S_{\infty}^{\pi+\varepsilon}$. 
TABLE I. The character table of the group $G_{48}$, the Complete Nuclear Permutation Inversion Group for methane $\left(\mathrm{CH}_{4}\right)$.

\begin{tabular}{lcccccccccc}
\hline & $E$ & $(123)$ & $(14)(23)$ & $(1423)^{*}$ & $(23)^{*}$ & $\mathrm{E}^{*}$ & $(123)^{*}$ & $(14)(23)^{*}$ & $(1423)$ & $(23)$ \\
& 1 & 8 & 3 & 6 & 6 & 1 & 8 & 3 & 6 & 6 \\
\hline $\mathrm{A}_{1}^{+}:$ & 1 & 1 & 1 & 1 & 1 & 1 & 1 & 1 & 1 & 1 \\
$\mathrm{~A}_{2}^{+}:$ & 1 & 1 & 1 & -1 & -1 & 1 & 1 & 1 & -1 & -1 \\
$\mathrm{E}^{+}:$ & 2 & -1 & 2 & 0 & 0 & 2 & -1 & 2 & 0 & 0 \\
$\mathrm{~F}_{1}^{+}:$ & 3 & 0 & -1 & 1 & -1 & 3 & 0 & -1 & 1 & -1 \\
$\mathrm{~F}_{2}^{+}:$ & 3 & 0 & -1 & -1 & 1 & 3 & 0 & -1 & -1 & 1 \\
$\mathrm{~A}_{1}^{-}:$ & 1 & 1 & 1 & 1 & 1 & -1 & -1 & -1 & -1 & -1 \\
$\mathrm{~A}_{2}^{-}:$ & 1 & 1 & 1 & -1 & -1 & -1 & -1 & -1 & 1 & 1 \\
$\mathrm{E}^{-:}:$ & 2 & -1 & 2 & 0 & 0 & -2 & 1 & -2 & 0 & 0 \\
$\mathrm{~F}_{1}^{-}:$ & 3 & 0 & -1 & 1 & -1 & -3 & 0 & 1 & -1 & 1 \\
$\mathrm{~F}_{2}^{-}:$ & 3 & 0 & -1 & -1 & 1 & -3 & 0 & 1 & 1 & -1 \\
\hline
\end{tabular}

TABLE II. The character table of $T_{\mathrm{d}}(\mathrm{M})$, the Molecular Symmetry Group for methane $\left(\mathrm{CH}_{4}\right)$.

\begin{tabular}{lccccc}
\hline & $E$ & $(123)$ & $(14)(23)$ & $(1423)^{*}$ & $(23)^{*}$ \\
\hline $\mathrm{A}_{1}:$ & 1 & 8 & 3 & 6 & 6 \\
$\mathrm{~A}_{2}:$ & 1 & 1 & 1 & 1 & 1 \\
$\mathrm{E}:$ & 1 & 1 & 1 & -1 & -1 \\
$\mathrm{~F}_{1}:$ & 2 & -1 & 2 & 0 & 0 \\
$\mathrm{~F}_{2}:$ & 3 & 0 & -1 & 1 & -1 \\
& 3 & 0 & -1 & -1 & 1 \\
\hline
\end{tabular}


TABLE III. The character table of $G_{36}$, the Molecular Symmetry Group for ethane $\left(\mathrm{C}_{2} \mathrm{H}_{6}\right)$.

\begin{tabular}{lllrrrrrrr}
\hline & $E$ & $(123)(456)$ & $(14)(26)(35)(78)^{*}$ & $(123)(465)$ & \multicolumn{2}{c}{$(132)$} & $(142635)(78)^{*}$ & $(14)(25)(36)(78)(142536)(78)$ & $(23)(56)^{*}$ \\
& 1 & 2 & 3 & 2 & 4 & 6 & 3 & 6 & 9 \\
\hline $\mathrm{A}_{1}:$ & 1 & 1 & 1 & 1 & 1 & 1 & 1 & 1 & 1 \\
$\mathrm{~A}_{2}:$ & 1 & 1 & 1 & 1 & 1 & 1 & -1 & -1 & -1 \\
$\mathrm{~A}_{3}:$ & 1 & 1 & -1 & 1 & 1 & -1 & 1 & 1 & -1 \\
$\mathrm{~A}_{4}:$ & 1 & 1 & -1 & 1 & 1 & -1 & -1 & -1 & 1 \\
$\mathrm{E}_{1}:$ & 2 & 2 & 2 & -1 & -1 & -1 & 0 & 0 & 0 \\
$\mathrm{E}_{2}:$ & 2 & 2 & -2 & -1 & -1 & 1 & 0 & 0 & 0 \\
$\mathrm{E}_{3}:$ & 2 & -1 & 0 & 2 & -1 & 0 & 2 & -1 & 0 \\
$\mathrm{E}_{4}:$ & 2 & -1 & 0 & 2 & -1 & 0 & -2 & 1 & 0 \\
$\mathrm{G}:$ & 4 & -2 & 0 & -2 & 1 & 0 & 0 & 0 & 0 \\
& & & & & & & & & 0 \\
\hline
\end{tabular}

TABLE IV. The character table of $D_{\infty \mathrm{h}}(\mathrm{M})$, the Molecular Symmetry Group for carbon dioxide $\left(\mathrm{CO}_{2}\right)$.

E $\quad(12) \quad E^{*} \quad(12)^{*}$

$\begin{array}{lllrrr}\text { (+s) } & \Sigma_{\mathrm{g}}^{+}: & 1 & 1 & 1 & 1 \\ (-\mathrm{a}) & \Sigma_{\mathrm{g}}^{-}: & 1 & -1 & -1 & 1 \\ (+\mathrm{a}) & \Sigma_{\mathrm{u}}^{+}: & 1 & -1 & 1 & -1 \\ (-\mathrm{s}) & \Sigma_{\mathrm{u}}^{-}: & 1 & 1 & -1 & -1\end{array}$

TABLE V. The character table of $D_{\infty \mathrm{h}}(\mathrm{EM})$, the Extended Molecular Symmetry Group for carbon dioxide $\left(\mathrm{CO}_{2}\right)$.

\begin{tabular}{cccccccccc}
\hline & & $E_{\mathrm{O}}$ & $E_{\varepsilon}$ & $\ldots$ & $\infty E_{\varepsilon}^{*}$ & $(12)_{\pi}^{*}$ & $(12)_{\pi+\varepsilon}^{*}$ & $\ldots$ & $\infty(12)_{\mathcal{E}}$ \\
& & 1 & 2 & $\ldots$ & $\infty$ & 1 & 2 & $\ldots$ & $\infty$ \\
\hline & & & & & & & & & \\
$(+\mathrm{s})$ & $\Sigma_{\mathrm{g}}^{+}:$ & 1 & 1 & $\ldots$ & 1 & 1 & 1 & $\ldots$ & 1 \\
$(-\mathrm{a})$ & $\Sigma_{\mathrm{g}}^{-}:$ & 1 & 1 & $\ldots$ & -1 & 1 & 1 & $\ldots$ & -1 \\
$(+\mathrm{a})$ & $\Sigma_{\mathrm{u}}^{+}:$ & 1 & 1 & $\ldots$ & 1 & -1 & -1 & $\ldots$ & -1 \\
$(-\mathrm{s})$ & $\Sigma_{\mathrm{u}}^{-}:$ & 1 & 1 & $\ldots$ & -1 & -1 & -1 & $\ldots$ & 1 \\
& $\Pi_{\mathrm{g}}:$ & 2 & $2 \cos (\varepsilon)$ & $\ldots$ & 0 & 2 & $2 \cos (\varepsilon)$ & $\ldots$ & 0 \\
& $\Pi_{\mathrm{u}}:$ & 2 & $2 \cos (\varepsilon)$ & $\ldots$ & 0 & -2 & $-2 \cos (\varepsilon)$ & $\ldots$ & 0 \\
& $\Delta_{\mathrm{g}}:$ & 2 & $2 \cos (2 \varepsilon)$ & $\ldots$ & 0 & 2 & $2 \cos (2 \varepsilon)$ & $\ldots$ & 0 \\
& $\Delta_{\mathrm{u}}:$ & 2 & $2 \cos (2 \varepsilon)$ & $\ldots$ & 0 & -2 & $-2 \cos (2 \varepsilon)$ & $\ldots$ & 0 \\
&. &. &. & $\ldots$ &. &. &. & $\ldots$ &. \\
&. &. &. & &. &. &. & &. \\
&. &. & & &. &. &. & &. \\
\hline
\end{tabular}

